\title{
Diabetes and Tuberculosis - Old Associates Posing a Renewed Public Health Challenge
}

\author{
Anil Kapur, ${ }^{1}$ Anthony D Harries, ${ }^{2}$ Knut Lönnroth, ${ }^{3}$ Ib C Bygbjerg ${ }^{4}$ and Pierre Lefèbvre ${ }^{5}$ \\ 1. Managing Director, World Diabetes Foundation; 2. Senior Advisor, International Union Against Tuberculosis and Lung Diseases; \\ 3. Medical Officer, Stop TB Department, World Health Organization; 4. Professor, Department of International Health, Medical Microbiology and Immunology,
}

University of Copenhagen; 5. Emeritus Professor of Medicine, University of Liège

DOI: $10.17925 / E E .2009 .05 .00 .10$

\section{Abstract}

Diabetes and tuberculosis (TB) have existed for thousands of years. Today, the global burden of disease from diabetes and TB is huge and, in the case of diabetes, rapidly increasing. Recent systematic reviews show that diabetes is associated with an increased risk of TB, yet the potential public health and clinical importance of the association seems to be largely ignored. Irrespective of whether the association is causal or a result of co-morbid factors, in low-resource societies with a dual disease burden, can a common health system approach for diabetes and TB be adapted to address prevention and care? How and to what extent can this be done? Good-quality implementation research is urgently needed to create robust action plans to address this double burden.
\end{abstract}

\section{Keywords}

Diabetes, tuberculosis (TB), co-morbidity, public health, clinical implications, millennium development goals (MDGs)

Disclosure: The authors have no conflicts of interest to declare.

Received: 23 April 2009 Accepted: 15 July 2009

Correspondence: Anil Kapur, World Diabetes Foundation, Lottenborgvej 24, 2800 Kgs Lyngby, Denmark. E: akap@worlddiabetesfoundation.org

\section{Intersecting Epidemics}

Diabetes and tuberculosis (TB) have existed for thousands of years. Great physicians in the ancient civilisations of Egypt, India, Greece and Rome described an illness that we now understand as diabetes. Similarly, the earliest evidence of TB has been found in the skeleton of a 30-year-old woman in Italy, dated to 5,800BCE. ' The term 'phthisis', or consumption, first appeared in Greek literature, and around 460BCE Hippocrates identified phthisis as the most widespread and invariably fatal disease of the times. The co-morbidity of diabetes and TB was also well known in those times. Richard Morton's Phthisiologia: or a treatise on consumption, written in 1694, stated that an association between the two conditions was suggested even in Roman times. ${ }^{2}$ The great Indian physician Susruta in about 600CE was aware of the association, and Avicenna in about 800CE commented that phthisis frequently complicated diabetes. ${ }^{3}$ Root, in reviewing the history of the association of diabetes and TB, noted that "in the latter half of the 19th century the diabetic patient appeared doomed to die of pulmonary TB if he succeeded in escaping coma". ${ }^{4}$ In 1883, Bouchardat stated "at autopsy every case of diabetes had tubercles in the lungs'..5 Indeed, half a century ago expert clinics were established for 'tuberculous diabetics' and appeared to be successful in reducing the otherwise high mortality rate. ${ }^{6}$

In the modern time, the global burden of disease from diabetes and TB is huge. According to the Diabetes Atlas published by the International Diabetes Federation (IDF), in 2007 there were an estimated 246 million people living with diabetes, amounting to $6 \%$ of the global adult population, and 308 million people with impaired glucose tolerance (IGT), amounting to $7.3 \%$ of the global adult population. There are 6-7 million new cases of diabetes and 3.5 million deaths ascribed to diabetes each year. In the same year (2007), it was estimated that there were 14.4 million people living with TB, 9.2 million new cases and 1.7 million deaths. ${ }^{7}$ While it is widely appreciated that $95 \%$ of TB patients live in the developing world, it is not so well known that $70 \%$ of diabetes patients also live in developing countries, especially in South-East Asia and the Western Pacific region. ${ }^{8}$ The number of adults with diabetes globally is likely to grow to an estimated 380 million by 2025 , and the majority of this increase will occur in low- and middle-income countries. Populous developing countries such as India, China, Brazil, the Russian Federation, Indonesia, Pakistan and Bangladesh rank quite highly in both the number of people with diabetes and the number of those with TB. Notable exceptions seem to be Mexico and Egypt, with a larger number of people with diabetes but a relatively lower ranking in terms of the number of people with TB, and Nigeria and South Africa, with a high ranking for the number of people with TB but a relatively lower ranking for the number of people with diabetes.

The incidence of TB is declining very slowly globally, at less than $1 \%$ annually. Speeding up the decline in incidence will require both scaling up of diagnostic and curative services (hopefully with the help of new tools that are now in the pipeline) and additional preventative actions, including addressing diabetes and other risk factors that increase the individual's susceptibility for TB. ${ }^{9}$ On the other hand, an increasing prevalence of diabetes may counteract the positive effects of improved curative services for TB.

In the face of this unprecedented health challenge, it is troubling that the potential public health and clinical importance of this relationship seems to be largely ignored and no significant initiative has been undertaken to jointly address this double burden. This neglect may have disastrous consequences. 


\section{Growing Evidence of a Causal Relationship}

There are several reports of high prevalence rates of diabetes in cases of pulmonary TB (PTB) (4-20\%), and rates are even higher for impaired glucose tolerance test (16-29\%). ${ }^{10} \mathrm{~A}$ recent systematic review that identified 13 relevant observational studies found that diabetes is associated with an increased risk of TB. ${ }^{11}$ Across the three cohort studies analysed, the pooled relative risk of TB associated with diabetes was 3.1 (95\% confidence interval $[\mathrm{Cl}] 2.27-4.26$ ), and in the case-control studies the odds ratios of TB ranged from 1.16 to 7.83 . The risks were higher in young people and in countries with a high background incidence of TB. Another systematic review reported similar findings, with relative risk estimates ranging from 1.5 to $7.8 .^{12}$ In India, with an estimated 21 million adults with diabetes and 900,000 incident PTB cases in 2000, diabetes accounted for nearly $15 \%$ of PTB and $20 \%$ of smear-positive PTB. ${ }^{13}$

The association is supported by the fact that diabetes patients have evidence of impaired cell-mediated immunity, micronutrient deficiency, pulmonary microangiopathy and renal insufficiency, all of which predispose to PTB. Innate and type 1 cytokine responses are higher in TB patients with associated diabetes than in non-diabetes control subjects. The effect is consistently and significantly more marked in diabetes patients with chronic hyperglycaemia. The impaired and altered immune response is also likely to increase susceptibility to infection with multidrug-resistant (MDR) strains..$^{14}$ The association could also be the result of non-enzymatic glycosylation of tissue proteins inducing alteration in bronchocilliary functions, or perhaps a result of diabetic autonomic neuropathy causing abnormal basal airway tone and consequent reduced bronchial reactivity and bronchodilation..$^{10}$ Although results are sometimes conflicting, several studies indicate that patients with TB who have diabetes present with a higher bacillary load in sputum, ${ }^{15,16}$ delayed mycobacterial clearance ${ }^{16,17}$ and higher rates of MDR infection..$^{18}$ Reviews of clinical studies ${ }^{13}$ show that diabetes patients with TB often present with lower lung infiltrates (similar to the radiographic pattern seen in patients with HIV/AIDS) and more cavitary lesions and may have worse treatment outcomes in terms of smear and culture conversion, case fatality and treatment failure. Recurrence or reactivation of previously treated TB with the onset of diabetes has been reported. A study among TB patients in south Texas and adjacent north-east Mexico ${ }^{19}$ reported that people with diabetes $27.8 \%$ in Texas and $20.0 \%$ in Mexico) were no more likely to have a history of previous TB than those without diabetes even after adjusting for age, gender and alcohol and drug abuse.

A recent study ${ }^{20}$ indicates that plasma levels of rifampicin may be considerably (53\%) lower in diabetes patients with TB compared with patients without diabetes. Perhaps the metabolism of rifampicin is affected by diabetes, rendering it less effective and predisposing to rifampicin resistance during treatment. Some investigators have reported an association between severity of TB and abnormal glucose tolerance.

Although the above data strongly suggest that diabetes is a causal risk factor for $T B$, the evidence is not conclusive. Some risk factors may predispose to both diabetes and $\mathrm{TB}$, e.g. tobacco smoking and alcoholism. It is also possible that at least part of the reported association is reversed, i.e. that the risk of diabetes is increased among people with TB. The stress due to a chronic infectious disease, such as $\mathrm{TB}$, that causes considerable catabolism may increase insulin resistance and increase the demand for insulin secretion. When the increased demand cannot be met (due to a pre-existing low $\beta$-cell mass), as is often the case in poor TB patients with associated malnutrition, the potential underlying risk of diabetes may be unmasked.

Regardless of the direction of the association, the common diabetes-TB co-morbidity presents clinical challenges: first as a result of stress-induced hyperglycaemia, second because rifampicin (one of the key drugs in any anti-TB regimen) may in itself have hyperglycaemic effects and third because of the interaction between rifampicin and several of the sulphonylurea group of oral hypoglycaemic agents, including gliclazide, glyburide, glipizide and glimepiride, which are metabolised by CYP2C9, a liver enzyme induced by rifampicin. The decreases in the area under the curve (AUC) for the various agents may range from 22 to $70 \%{ }^{21}$ and require dose adjustment. Also important is the fact that isoniazid (INH), another important and common drug used to treat $T B$, is prone to causing peripheral neuropathy, which may worsen or mimic diabetic peripheral neuropathy; vitamin $B_{6}$ supplementation may be necessary. Pyrazinamide may interfere with urine ketone testing. On theoretical grounds, it is also possible that people with diabetes need a different TB treatment regimen due to higher risk of treatment failure.

\section{Public Health and Clinical Implications of the Apparent Association Between Diabetes and Tuberculosis}

Irrespective of whether the relation between diabetes and TB is causal or caused by joint co-factors, in societies with relatively few resources for healthcare and a double burden of non-communicable and communicable diseases, management and control may benefit from an integrated approach. How and to what extent can this be done?

\section{Screening for Diabetes Among Tuberculosis Patients?}

Should all TB patients be screened for diabetes? A clinical study in Tanzania, with an already high threshold for recognising diabetes, showed that unless an oral glucose tolerance test was performed at the start of therapy, over half of the cases with diabetes would have been missed. ${ }^{22}$ In developing countries awareness of diabetes is generally low, and because people with type 2 diabetes may have no or limited symptoms it is quite likely that poor and less educated patients will give no history suggestive of diabetes, particularly in the presence of symptomatic TB. ${ }^{23}$ Screening for diabetes is thus the only sure way to rule out concomitant disease. It may therefore be of value to screen TB patients for diabetes, but what is the most cost-effective means of doing this? While we gather data to address these questions, simple and economically realistic approaches can be immediately implemented at every TB clinic worldwide. These include documenting self-reported diabetes in every new patient with TB and, where feasible, performing a fingerstick glucometer assay for blood glucose. Patients with high readings can then be flagged for potential treatment failure and be accorded special attention. ${ }^{24}$

\section{Screening for Tuberculosis Among Diabetes Patients?}

Should every patient with diabetes be screened for latent TB infection, at least in countries with a high TB burden? Current screening tests are challenging to use in resource-limited settings, and include the intradermal injection of tuberculin, which is read 48-72 hours later, or the more expensive interferon-gamma release assays (IGRAS), which are costly and require specific laboratory equipment. If these results are positive, how should the progression 
of latent infection to disease be monitored or what should be done to prevent TB? Should these patients be given a course of isoniazid preventative therapy? Should patients with TB and diabetes be treated with a different drug regimen from the regimen used to treat those with TB alone? Simple screening algorithms currently used in HIV-infected patients in high HIV-TB burden countries evaluate patients for cough, fever, night sweats, weight loss and chest pain. A positive response indicates the need for further assessment by sputum smear examination and chest radiography. The value of such screening tools in the diabetes clinics of developing countries is not known. ${ }^{8}$ Moreover, sputum smear is highly insensitive; given the propensity of people with diabetes for reactivation, do negative smears in people with diabetes have the same significance as in those without diabetes? Finally, in most developing countries there are no systematic ways of monitoring or evaluating patients with noncommunicable diseases. This has to change. The directly observed treatment short-course (DOTS) framework for TB control, which was developed by the International Union against Tuberculosis and Lung Disease and WHO, has allowed structured, well-monitored services to be delivered to millions of TB patients in some of the poorest countries in the world. This model can be adapted for noncommunicable diseases such as diabetes. ${ }^{25}$

\section{Need for Action}

There is a widely held misconception among policy-makers, multilateral donors and even public health experts, particularly in the developed world, that diabetes is a rich man's disease. However, the fact is that the low- and middle-income countries undergoing rapid urbanisation are witnessing the fastest growth in diabetes rates, and the worst affected are the urban poor in these countries. In this context diabetes shares many risk factors and socioeconomic determinants of poor health outcomes with TB, and may therefore be found in the same subpopulations. In most developing and low-resource countries there are very few healthcare resources available for diabetes prevention and care. Can a common health system approach to diabetes and TB be adapted to address prevention and care?

Millennium Development Goal 6, target 8, specifies that the incidence of infectious diseases such as TB should be halted and reversed by
2015. The goal for TB elimination is a global incidence of less than one case per million by 2050. To succeed in achieving these targets, in resource-poor countries it is important to focus not only on improved access to TB diagnosis and treatment and on HIV/AIDS, but also on the burgeoning epidemic of diabetes as a significant epidemiological risk factor. The link between TB and diabetes has been established; what is needed now is good-quality implementation research to screen for, care for, prevent and monitor this dual burden of disease.

Anil Kapur is Managing Director of the World Diabetes Foundation. He specialises in internal medicine and has written books on diabetes for both medical professionals and lay people, and has published more than 60 papers in international journals in the areas of internal medicine, clinical pharmacology and diabetes. Dr Kapur has co-ordinated several large studies, including the DiabCare Asia India study, Cost of Diabetes Care in India and the National Urban Diabetes Survey.

Anthony D Harries is Senior Advisor at the International Union Against Tuberculosis and Lung Disease and an Honorary Professor at the London School of Hygiene and Tropical Medicine. He worked in Malawi for 20 years, where he held the posts of physician, Foundation Professor of Medicine, National Technical Advisor to the Malawi Tuberculosis Control Programme and National Advisor in HIV Care and Support at the Ministry of Health.

Knut Lönnroth is a Medical Officer in the Tuberculosis Department at the World Health Organization (WHO), where he is the focal person for work on tuberculosis risk factors and social determinants. He is a medical doctor with specialisation in social medicine, and has a PhD in public health. Dr Lönnroth is involved in international health research with a focus on health systems and social factors, particularly in relation to tuberculosis control.

Ib C Bygbjerg is a Professor in the Department of International Health, Medical Microbiology and Immunology at the University of Copenhagen. He teaches international health, trains healthcare professionals and conducts research in Denmark, Ghana, India, Tanzania and Vietnam. Professor Bygbjerg founded the Centre for International Health and Development and Cluster in International Health, based at the University of Copenhagen, which carries out research in diabetes, HIV/AIDS, tuberculosis, malaria and their interactions.

Pierre Lefèbvre is Emeritus Professor of Medicine, Past Chairman of Medicine and Head of the Division of Diabetes, Nutrition and Metabolic Disorders at the University of Liège. His research interests include physiology and pathophysiology of metabolic regulations in type 2 diabetes and obesity. He serves as Chairman of the Board of Directors of the World Diabetes Foundation and was President of the International Diabetes Federation from 2003-2006.
1. Canci A, Minozzi S, Borgognini Tarli S, New evidence of tuberculous spondylitis from Neolithic Liguria (Italy), Int $\mathrm{J}$ Osteoarchaeol, 1996;6:497-501

2. Dixon B, Diabetes and tuberculosis: an unhealthy partnership. Availablle at: infection.thelancet.com

3. Gupta A, Shah A, Tuberculosis and diabetes: an appraisal, Ind I Tub, 2000;47:3.

4. Root HF, The association of diabetes and tuberculosis, N Engl J Med, 1934;1:210.

5. Younger D, Hadley WB. In: Marble A, White P, Bradley RF, Krall LP (eds), Joslin's diabetes mellitus. 11th ed, Philadelphia: Lea and Febiger, 1971;628-31.

6. Luntz G, Tuberculous diabetics: the Birmingham Regional Service, Lancet, 1954;266(6819):973-4.

7. Global TB Control, 2009, Geneva: World Health Organization, 2009.

8. Harries AD, Billo N, Kapur A, Links between diabetes mellitus and tuberculosis: should we integrate screening and care?, Trans R Soc Trop Med Hyg, 2009;103:1-2

9. Lönnroth K, Raviglione M, Global Epidemiology of Tuberculosis: Prospects for Control, Semin Respir Crit Care Med, 2008;29:481-91.

10. Kant $L$, Diabetes Mellitus-Tuberculosis: The Brewing Double Trouble, Indian J Tuberc, 2003:5:183-4.

11 .Jeon $\mathrm{CY}$, Murray MB, Diabetes mellitus increases the risk of active tuberculosis: a systematic review of 13 observational studies, PLOS Med, 2008;5:e152.

12. Stevenson CR, Forouhi NG, Roglic G, et al., Diabetes and tuberculosis: the impact of the diabetes epidemic on tuberculosis incidence, BMC Public Health, 2007;7:234.

13. Stevenson CR, Critchley JA, Forouhi NG, et al., Diabetes and the risk of tuberculosis: a neglected threat to public health, Chronic IIIn, 2007;3:228-45.

14. Restrepo BI, Fisher-Hoch SP, Pino PA, et al., Tuberculosis in poorly controlled type 2 diabetes: altered cytokine expression in peripheral white blood cells, Clin Infect Dis, 2008;47(5):634-41.

15. Restrepo BI, Fisher-Hoch SP, Crespo JG, et al., Type 2 diabetes and tuberculosis in a dynamic bi-national border population, Epidemiol Infect, 2007;135:483-91.

16. Singla $\mathrm{R}$, Khan $\mathrm{N}$, Al-Sharif $\mathrm{N}$, et al., Influence of diabetes on manifestations and treatment outcome of pulmonary TB patients, Int J Tuberc Lung Dis, 2006;10:74-9.

17. Guler M, Unsal E, Dursun B, et al., Factors influencing sputum smear and culture conversion time among patients with new case pulmonary tuberculosis, Int I Clin Pract, 2007;61:231-5.

18. Bashar M, Alcabes P, Rom WN, Condos R, Increased incidence of multidrug-resistant tuberculosis in diabetic patients on the Bellevue Chest Service, 1987 to 1997,
Chest, 2001;120:1514-19.

19. Fisher-Hoch SP, Whitney E, Mccormick JB, et al.; and The Nuevo Santander Tuberculosis Trackers, Type 2 diabetes and multidrug-resistant tuberculosis, Scand I Infect Dis, 2008;1-6:l.

20. Nijland HM, Ruslami R, Stalenhoef JE, et al., Exposure to rifampicin is strongly reduced in patients with tuberculosis and type 2 diabetes, Clin Infect Dis, 2006;43:848-54.

21. Park JY, Kim KA, Park PW, et al., Effect of rifampin on the pharmacokinetics and pharmacodynamics of gliclazide, Clin Pharmacol Ther, 2003;74:334-40.

22. Mugusi F, Swai AB, Alberti KG, McLarty DG, Increased prevalence of diabetes mellitus in patients with pulmonary tuberculosis in Tanzania, Tubercle, 1990;71: 271-6.

23. Surya Kirani KRL, Santha Kumari V, Lakshmi Kumari R, Co-existence of pulmonary tuberculosis and diabetes mellitus: some observations, Ind J Tub, 1998;45:47-8.

24. Restrepo $\mathrm{Bl}$, Convergence of the Tuberculosis and Diabetes Epidemics: Renewal of Old Acquaintances, Clin Infect Dis, 2007;45:436-8

25. Harries AD, Jahn A, Zachariah R, Enarson D, Adapting the DOTS framework for tuberculosis control to the management of non-communicable diseases in subSaharan Africa, PLoS Med, 2008;5:e124. 